\title{
Cues of intraguild predators affect the distribution of intraguild prey
}

\author{
Yasuyuki Choh · Tessa van der Hammen • \\ Maurice W. Sabelis $\cdot$ Arne Janssen
}

Received: 6 October 2009 / Accepted: 3 March 2010 / Published online: 31 March 2010

(C) The Author(s) 2010. This article is published with open access at Springerlink.com

\begin{abstract}
Theory on intraguild (IG) predation predicts that coexistence of IG-predators and IG-prey is only possible for a limited set of parameter values, suggesting that IG-predation would not be common in nature. This is in conflict with the observation that IG-predation occurs in many natural systems. One possible explanation for this difference might be antipredator behaviour of the IG-prey, resulting in decreased strength of IG-predation. We studied the distribution of an IG-prey, the predatory mite Neoseiulus cucumeris (Acari: Phytoseiidae), in response to cues of its IG-predator, the predatory mite Iphiseius degenerans. Shortly after release, the majority of IG-prey was found on the patch without cues of IG-predators, suggesting that they can rapidly assess predation risk. IG-prey also avoided patches where conspecific juveniles had been killed by IG-predators. Because it is well known that antipredator behaviour in prey is affected by the diet of the predator, we also tested whether IG-prey change their distribution in response to the food of the IG-predators (pollen or conspecific juveniles), but found no evidence for this. The IG-prey laid fewer eggs on patches with cues of IG-predators than on patches without cues. Hence, IG-prey changed their
\end{abstract}

Communicated by Sven Bacher.

Y. Choh · T. van der Hammen · M. W. Sabelis · A. Janssen ( $\square)$ Section of Population Biology, Institute for Biodiversity and Ecosystem Dynamics, University of Amsterdam, Science Park 904, 1098 XH Amsterdam, The Netherlands e-mail: arne.janssen@uva.nl

Present Address:

Y. Choh

Center for Ecological Research, Kyoto University,

Otsuka 509-3, Hirano, Kamitanakami, Otsu 520-2113, Japan distribution and oviposition in response to cues of IG-predators. This might weaken the strength of IG-predation, possibly providing more opportunities for IG-prey and IG-predators to co-exist.

Keywords Ideal free distribution - Intraguild predation . Predator avoidance $\cdot$ Predator-prey interaction . Phytoseiid mites

\section{Introduction}

Intraguild predation (IGP) is the killing and eating of prey species by a predator that can also utilise the resources of those prey (Polis et al. 1989). Hence, the predator and its prey are also potential competitors. IGP is a special case of omnivory, which is defined as feeding on resources at different trophic levels. Theory on IGP predicts possible coexistence of all three species, including the resource, only if the intermediate consumer (intraguild prey, IG-prey, hereafter) is superior to the other consumer (IG-predator) in competing for the shared resource (Holt and Polis 1997). Even then, the parameter space in which this coexistence can occur is limited to intermediate levels of productivity (Mylius et al. 2001). Hence, theory predicts that IGP would not be common in nature (Janssen et al. 2007), which contradicts empirical observations (Polis et al. 1989; Polis and Holt 1992; Holt and Polis 1997; Rosenheim et al. 1995; Polis and Winemiller 1996). This conflict between theory and empirical evidence might be due to the fact that theory so far did not take habitat structure and antipredator behaviour into account, and these factors may reduce the strength of IGP and thereby increase the parameter space allowing for coexistence (Heithaus 2001; Janssen et al. 2007). For example, prey may use habitat structure as a refuge, 
resulting in the reduced rate of encounter between predator and prey (Persson and Eklov 1995). In addition to refuge use, habitat selection by the prey may also reduce the encounter rate between IG-predators and IG-prey.

It is well known that various species of IG-prey show antipredator behaviour in the presence of predators or predator-related cues (Faraji et al. 2000; Venzon et al. 2000; Persons and Rypstra 2001; Agarwala et al. 2003; Wilder and Rypstra 2004; Magalhães et al. 2005; Nakashima et al. 2006; Montserrat et al. 2007; Rypstra et al. 2007). For example, IG-prey may avoid odours of IG-predators, suggesting that they recognize IG-predators from a distance (Venzon et al. 2000; Magalhães et al. 2005). Faraji et al. (2000) showed that IG-prey avoid ovipositing near eggs of IG-predators and tend to displace eggs of IG-predators more frequently than conspecific eggs. If IG-prey avoid patches with IG-predators, this might reduce the strength of IGP, resulting in increased possibilities for coexistence of IG-prey and IG-predators. We therefore studied the distribution of IG-prey in response to the presence of (cues of) IG-predators.

We specifically assessed whether differences in perceived predation risk affect the distribution of IG-prey. We used two predatory mite species, Iphiseius degenerans and Neoseiulus cucumeris. The predatory mites have overlapping distributions in the Mediterranean area (De Moraes et al. 2004), both feed on pollen and thrips (van Rijn and Tanigoshi 1999) and are reciprocal IG-predators of each others' juveniles. The per capita attack rate of adult $I$. degenerans on juvenile $N$. cucumeris is much higher than that of adult $N$. cucumeris on juvenile $I$. degenerans (Montserrat et al. 2006), suggesting that $I$. degenerans is a stronger IG-predator than $N$. cucumeris. Here, we consistently refer to $N$. cucumeris as "IG-prey" and I. degenerans as "IG-predator" to simplify the text and facilitate its interpretation. We examined the effect of the risk of IGP on the distribution of female adult IG-prey, focusing on the potential cues related to IGP.

\section{Materials and methods}

Mite cultures and experimental conditions

The origin of the strains of I. degenerans and N. cucumeris and the rearing methods are described by van Rijn and Tanigoshi (1999). Iphiseius degenerans was reared on large rectangular PVC arenas $(35 \times 20 \mathrm{~cm})$ placed on top of a 4-cm-high foam pad in a larger water-containing plastic utility tray. To provide a water source for the mite colony, the edges of the arena were covered with wet tissue paper that touched the water barrier in the tray. For additional water sources, three strips of moist filter paper were placed across the arena at equal distances. Sewing threads served as oviposition substrates. The cultures were provided twice per week with birch pollen (Betula pubescens Ehrh.) as food (van Rijn and Tanigoshi 1999). Neoseiulus cucumeris was kept on smaller rectangular plastic arenas $(8 \times 15 \mathrm{~cm})$. The edges of the arenas were also covered with tissue paper that was in contact with the water. They were fed Typha sp. pollen twice per week (van Rijn and Tanigoshi 1999).

All females used in the experiments were 10- to 14-daysold since egg deposition. Experiments were carried out in a climate room at $25 \pm 1{ }^{\circ} \mathrm{C}, 16: 8 \mathrm{~h} \mathrm{~L} / \mathrm{D}$, and $60 \pm 5 \mathrm{RH}$.

\section{Experimental set-up}

Two acrylic plates $(15 \times 8 \mathrm{~cm})$ were used as patches for predatory mites. Black plastic sheets were placed on top of sponges in water-containing trays, and the acrylic plates were put on top of these plastic sheets. The sheets facilitated the detection of the light-coloured IG-prey. The edges of the plates were covered with tissue paper that was in contact with the water, thus serving both as barrier and water source. One acrylic plate (patch) was treated, the other was used as control. The two plates were interconnected with yellow plastic tape $(2 \mathrm{~cm}$ length, $3 \mathrm{~cm}$ width), serving as a bridge, allowing the mites to cross from one patch to the other. Almost all mites were found on either of the patches. The mites on the bridge were assigned to the closest patch. Ample amounts of Typha sp. pollen were placed at the centre of each patch as a food source.

\section{Distribution of IG-prey between two patches}

To test effects of the risk of IGP on the distribution of IG-prey, we treated one of the two patches by providing them with cues left by IG-predators prior to the experiment: (1) 25 IG-predators that were not supplied with any food, hence that were starved during the treatment of the patch, (2) 25 IG-predators that were supplied with Typha sp. pollen, (3) 25 IG-predators and 50 juvenile IG-prey, (4) 2 IG-predators and 50 juvenile IG-prey, or (5) 50 juvenile IG-prey. The mites were introduced to these patches, which also contained a polyester thread $(3 \mathrm{~cm})$ as a site for oviposition. We expected that the mites left cues related to IG-predators and IGP, and kept the mites on the treated patches for $24 \mathrm{~h}$. The mites and their eggs, as well as the thread, were removed from the patch $24 \mathrm{~h}$ after the introduction. Other predator cues, such as faeces, and dead juvenile IG-prey in case these were present, were left on the patch. Subsequently, an ample amount of pollen was placed in the centre of the patches except for treatment with IG-predators that had been supplied with pollen, because ample pollen was still present on the patch (treatment 2). In all cases, the 
alternative patch was a patch that was not exposed to predators, containing the same amount of Typha sp. pollen as the predator-exposed patch. After one of the two patches was treated as described above, it was connected to a clean patch with yellow plastic tape, serving as a bridge, and 150 adult female IG-prey were placed on the bridge and their distribution was observed for the next $48 \mathrm{~h}$. As we released the same number of IG-prey in all experiments, we were able to test how previous exposure of a patch to differential predation risk affects the distribution of prey. If only the availability of food would determine this distribution and not cues of predators, the mites were expected to distribute themselves equally over both patches. As a further test for this, (6), IG-prey were offered two clean patches with Typha sp. pollen.

In treatment 1 , the effect of the previous presence of IG-predators on the distribution of IG-prey was examined. We tested whether the previous diet of IG-predators that were used to treat the patch affected the distribution of IG-prey by comparing the treatments 1,2 , and 3 . To test whether differences in the risk of IGP affected the distribution of IG-prey, we prepared patches that had contained a higher density (treatment 3 ) or a lower density (treatment 4) of IG-predators. In treatment 5, we examined whether IG-prey preferred a patch with juvenile conspecifics to a clean patch. This is the control experiments for treatments 3 and 4. IG-prey crossed the bridge between patches on average $13.2 \pm 0.57$ times in $48 \mathrm{~h}$, showing that the mites could easily cross the bridge and hence freely move between the two patches (van der Hammen et al., in preparation).

To avoid disturbing the mites, they were not counted under a binocular microscope, but the entire patches were photographed after $10 \mathrm{~min}$ and 2, 4, 6, 24, 26, 28, 30, $48 \mathrm{~h}$ (Sony Cyber-shot DSC-W15, 5.1 Mega pixels, Japan). The numbers of mites on each patch were counted from the photographs (van der Hammen et al., in preparation). As it took some time $(10 \mathrm{~min})$ to take a picture after introducing the mites on the patch, we refer to the distribution seen on the pictures as that at $10 \mathrm{~min}$. The numbers of mites counted on the pictures were strongly correlated with the numbers counted using a dissecting microscope $\left(R^{2}=0.71, d f=58\right.$, $P<0.0001$, Pearson's product-moment correlation). The number of IG-prey eggs on each patch was counted $48 \mathrm{~h}$ after the introduction under a microscope (magnification $\times 10)$. We repeated each treatment ten times $\left(25 \pm 1{ }^{\circ} \mathrm{C}\right.$, 16:08 h L/D, and $60 \pm 5 \mathrm{RH})$. Because it was impossible to do all experiments at the same time, we randomised the time at which they were performed, taking care that all treatments were performed during the same period. The position of the treated patch was changed between replicates to correct for potential asymmetries in the experimental set-up or environment.
Statistical analysis

Within each experiment, we first tested whether the distribution of the mites over the two patches changed with time using a linear mixed effects model (lme of the library nlme of R; R Development Core Team 2008), corrected for repeated measures and the arcsine square root transformed fraction of mites on the treated patch as dependent variable. As we found no effect of time on the distributions in a large majority of the treatments, we subsequently tested whether the mites were distributed randomly over the two patches at $10 \mathrm{~min}$ after the introduction. To this end, we compared the fraction of mites on the treated patch with an expected fraction of 0.5 using a $t$ test, after having checked the fractions for normality both visually and with a Shapiro-Wilk normality test (Crawley 2007). The distribution of eggs within each treatment was tested using the same procedure. In addition, the distributions of adult mites at $10 \mathrm{~min}$ after the introduction, as well as the distributions of eggs at the end of the experiments, were compared among treatments with a generalized linear model with a quasibinomial error distribution to correct for overdispersion, followed by a posteriori contrasts through model simplification (Crawley 2007; R Development Core Team 2008).

\section{Results}

The distribution of adult female IG-prey changed over time in only two of the six treatments; the treatment with cues of 25 starved IG-predators (lme, $F_{1,79}=7.10, P=0.0093$; Fig. 1) and the treatment with cues of 25 IG-predators in the presence of pollen (lme, $F_{1,79}=9.15, P=0.0034$; Fig. 1). In these two treatments, the initial distribution was more strongly skewed than the final distribution. This was possibly caused by waning of the cues left by the IG predators with time. We therefore decided to compare the distributions of IG-prey between two patches, soon after the introduction of IG-prey (10 min).

The initial distributions of IG-prey differed significantly among treatments (Fig. 2; GLM, $F_{5,54}=4.2, P=0.0027$ ). Without any predator cues (control), adult female IG-prey were distributed evenly over the two patches (Figs. 1 and 2). With cues left by 25 starved or fed IG-predators, a larger fraction of IG-prey was found on the untreated patches than on the treated patches (Figs. 1 and 2). Moreover, the proportions of IG-prey on the patches that had contained 25 IG-predators in the three treatments were significantly lower than that on a patch in the control treatment, irrespective of the presence of 50 juvenile IG-prey (Fig. 2). A smaller fraction of IG-prey was also found on the patch with cues from 25 IG-predators plus 50 conspecific juveniles (Figs. 1 and 2). With 2 IG-predators plus 50 juvenile 


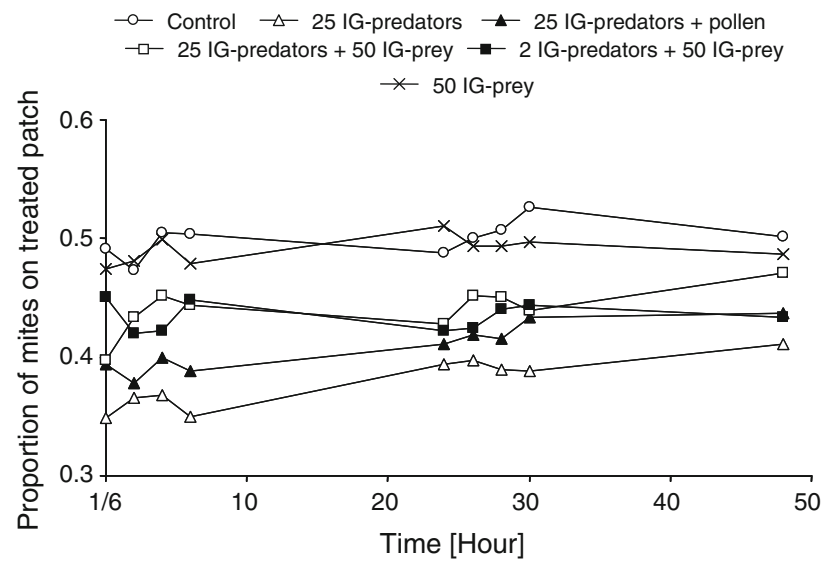

Fig. 1 The fraction of IG-prey on the treated patches from $10 \mathrm{~min}$ $(1 / 6 \mathrm{~h})$ to $48 \mathrm{~h}$ after their introduction. The distribution of IG-prey was measured on two interconnected clean patches (control), or a clean patch interconnected with a patch with cues left by IG-predators. IG-predators were either released on one of the two patches without food (25IG-predators), with pollen as food (25IG-predators + pol$l e n)$, or with juvenile IG-prey as food (25IG-predators + 50 IG-prey and 2 IG-predators + 50 IG-prey). As a final control, a patch was treated by releasing 50 juvenile IG-prey without IG-predator (50 $I G$-prey). A random distribution of the IG-prey over the two patches corresponds to a proportion of 0.5 of all mites on the treated patch. For reasons of clarity, standard errors are not given

$\square$ Control $\square 25$ IG-predators

25 IG-predators + pollen 25 IG-predators + 50 IG-prey 2 IG-predators + 50 IG-prey एس 50 IG-prey

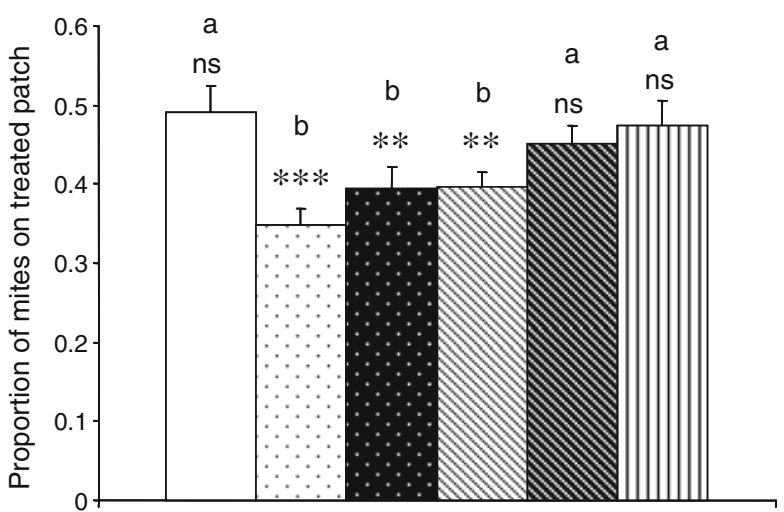

Fig. 2 The initial proportion (+SEM) of IG-prey on the treated patch. See legend to Fig. 1 for further explanation. For each treatment, the observed distributions were tested against a random distribution (proportion of 0.5 ) with a $t$ test: $n s$ not significant; $P>0.05 ; * * P<0.005$; $* * * P<0.0001$. Different letters above each bar indicate significant differences among the treatments (GLM, $P<0.0001)$

IG-prey, no such effect was observed (Figs. 1 and 2). The proportions of IG-prey on the patches treated with 25 IG-predators plus 50 conspecific juveniles differed significantly from that on the patches with cues of 2 IG-predators plus 50 conspecific juveniles (Fig. 2). This is likely due to the lower concentration of cues associated with two rather
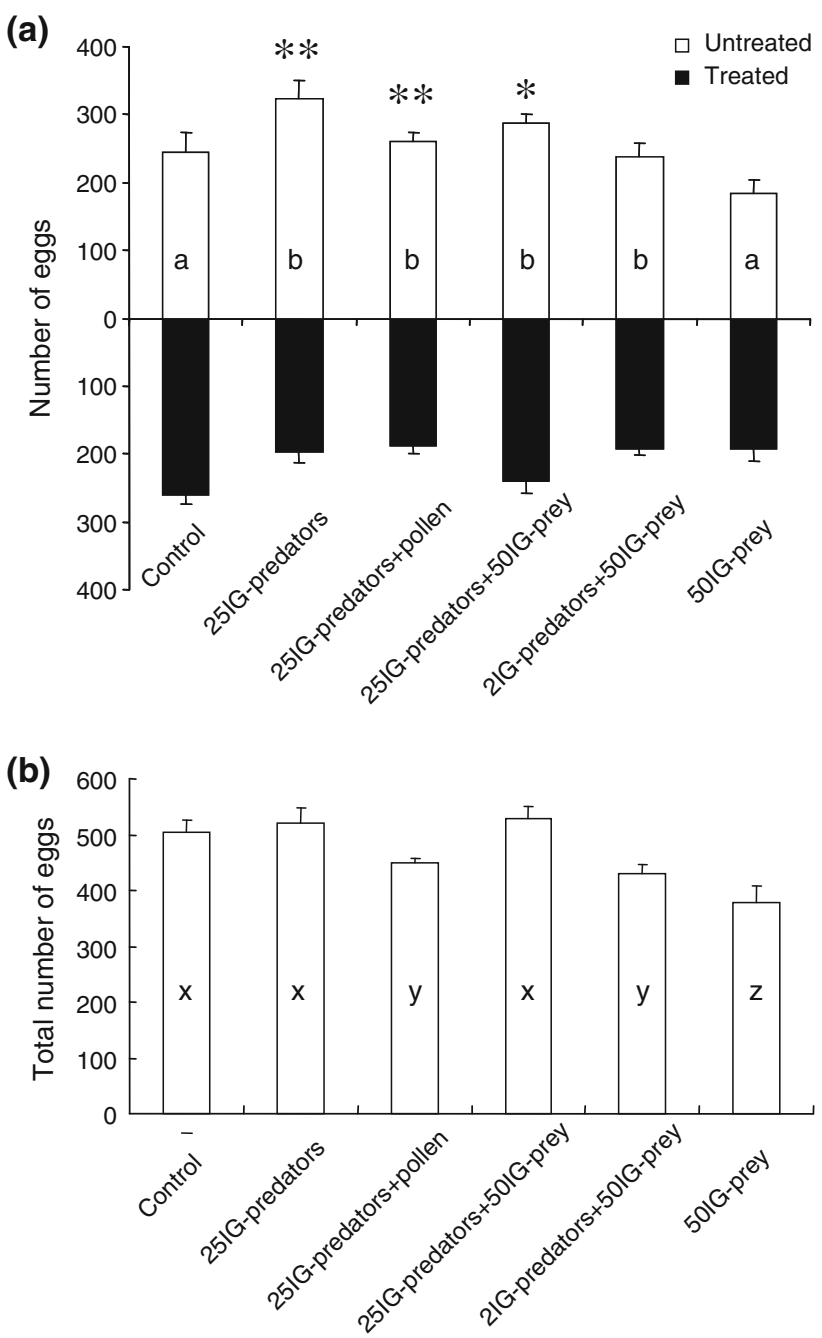

Fig. 3 The distribution and total number of eggs laid by IG-prey during $48 \mathrm{~h}$. The eggs of IG-prey were counted on two connected clean patches (control), or a clean patch connected to a patch treated with IG-predators. See legend to Fig. 2 for further explanation. a The distribution of the eggs over the two patches per treatment. Shown are the average number (+SEM) of eggs on the treated (black bars extending downwards) and untreated patch (white bars extending upwards). Asterisks indicate significant differences in the oviposition between the clean patch and the treated patch within each treatment: $* P<0.05$, $* * P<0.01$. Differences in distribution of eggs among treatments are indicated with different letters inside the bars. $\mathbf{b}$ The average (+SEM) total number of eggs on both patches per treatment. Differences in total numbers of eggs among treatments are indicated with different letters inside the bars

than 25 IG-predators. Likewise, no surviving juvenile IG-prey were found in the treatment with 25 IG-predators, but on average $5.0 \pm 0.54$ (mean $\pm \mathrm{SE}$ ) surviving juveniles were found in the treatment with 2 IG-predators. The presence of only 50 juvenile IG-prey, without IG-predators, did not have an effect on the distribution of IG-prey (Figs. 1,2).

There was a significant effect of treatment on the distribution of eggs of the IG-prey (Fig. 3a; GLM, $F_{5,54}=3.38$, $P=0.01)$. The distribution of eggs differed significantly 
among the treatments with and without cues from IG-predators (GLM, $F_{1,59}=12.2, P=0.0009$; Fig. 3a). A larger proportion of eggs of IG-prey was found on the untreated patch than the treated patch in three treatments (Fig. 3a); the treatment with cues from 25 IG-predators ( $t$ test, $\left.t_{9}=3.97, P=0.003\right)$, the treatment with cues from 25 IG-predators with pollen $\left(t_{9}=3.53, P=0.006\right)$, and the treatment with cues from 25 IG-predators plus 50 IG-prey juveniles $\left(t_{9}=2.46, P=0.036\right)$. Furthermore, there was a significant effect of treatment on the total number of eggs produced (hence, on both patches together) (Fig. 3b; $\left.F_{5,54}=6.74, P<0.0001\right)$.

\section{Discussion}

The distribution of IG-prey over two patches did not significantly change with time following the introduction in most treatments on the two-patch systems. Moreover, the IG-prey clearly settled less on patches with cues of the IG-predator. These findings suggest that IG-prey can perceive patch quality quickly, using cues related to IG-predators to avoid patches with an increased risk of IGP. Much theory on searching and distributions of foragers assumes that animals have perfect knowledge of their environment (Fretwell and Lucas 1970; Fretwell 1972), but there is also general agreement that this assumption is unrealistic. Our findings show that foraging animals can use cues to rapidly assess patch quality, which may bring them close to behaving as omniscient foragers at the spatial scale studied in our experiments (Janssen et al. 1997). We do not know how exactly the IG-prey were able to quickly assess patch quality. Cues of IG-predators were present on the entire treated patches, so IG-prey could possibly perceive cues upon entering the patch. Because they can quickly move from one patch to the other (van der Hammen et al., in preparation), this would allow the mites to rapidly settle on a patch without cues associated with predators. Alternatively, some of the cues may have been volatile, allowing the mites to assess patch quality from a distance (Magalhães et al. 2005), which would also result in the mites reaching their final distributions rapidly. Further studies are needed to clarify the nature of the cues (e.g., volatile or not).

Several studies have reported that prey avoid areas with higher predation risk (Stephens and Krebs 1986; Lima and Dill 1990; Lima 1998; Sih 1998, Magalhães et al. 2005). IG-prey may use faeces of IG-predators (Agarwala et al. 2003) and semiochemicals in trails of IG-predators (Persons and Rypstra 2001; Nakashima et al. 2006) as cues for avoidance of IGP. Although the distribution of IG-prey changed over time only when cues of IG-predators were present on the treated patch (Fig. 1), the distribution did not change over time when there were cues from both IG-predators and juvenile IG-prey on the treated patch (Fig. 1). This suggests that cues of IG-predators on the treated patch might fade away faster than cues associated with dead conspecific juveniles, or that the latter cues induce a more persistent antipredator response in the adult IG-prey.

In this study, more IG-prey were found on clean patches than on patches with cues of IG-predators. It is known that the previous diet of IG-predators can affect antipredator behavior in IG-prey (Venzon et al. 2000; Magalhães et al. 2005). However, the distribution of IG-prey did not change with the previous diet of the IG-predators (Fig. 2). Furthermore, there were significant differences in the distribution with cues of 25 IG-predators and with cues of 2 IG-predators or no IG-predators (Fig. 2). This suggests that the response of IG-prey depends, at least to some extent, on the concentration of IG-predator cues. Interestingly, it seems that the cues of intraguild predation did not induce strong antipredator behaviour compared to the cues of IG-predators.

We found that adult female IG-prey oviposited more in the absence of cues of IG-predators than on patches with such cues (Fig. 3a). Although there were no significant differences in the oviposition on the treated and untreated patches when there were cues from both 2 IG-predators and 50 conspecific juveniles on the treated patch (Fig. 3a), there was also a tendency to lay more eggs on the cue-free patches in this treatment. Furthermore, the distribution pattern of eggs of IG-prey differed among treatments with and without IG-predators (Fig. 3a). Using the same species of predatory mites, Montserrat et al. (2007) reported that the IG-prey retain their eggs in the presence of IG-predators, but not when exposed to cues left by the predators. Because the IG-prey in our study could only perceive cues of the IG-predators, this suggests that IG-prey did not retain eggs but instead changed the oviposition pattern over two patches in response to cues of IG-predators.

As adult IG-prey are not fed upon by adult IG-predators, the adult IG-prey might change the distribution between two patches to avoid laying eggs near IG-predators and thus to reduce predation risk for their offspring. In addition to the risk of IGP, the lower numbers of IG-prey on patches with cues of IG-predators might be explained by avoidance of competition for food. The total number of eggs of IG-prey was lowest when offered a treated patch with cues of 50 conspecific juveniles (Fig. 3b), suggesting that IG-prey might reduce oviposition in the presence of cues of conspecifics to reduce competition. These explanations are not mutually exclusive.

The theory on IGP predicts that IG-prey and IG-predators can coexist when the IG-prey is superior at exploitative competition for the shared resource, whereas the IG-predator compensates for this lack of competitiveness by 
consuming the IG-prey (Holt and Polis 1997). Recently, a theoretical (HilleRisLambers and Dieckmann 2003) and experimental paper (Montserrat et al. 2008) showed that the possibilities for coexistence of IG-predators and IG-prey involved in reciprocal intraguild predation are even more restricted that in systems with simple IGP. Instead, the predator that settles first can prevent the other predator species from invading (priority effect). Here, we show that the IG-prey avoid patches that had previously been exposed to the IG-predators, resulting in reduced oviposition on the patch (van der Hammen et al., in preparation). The distribution of the IG-prey and their eggs will be an important factor determining the initial conditions of populations of the IG-prey. Furthermore, the IG-predator is reported to oviposit more frequently near conspecific eggs than near eggs of the IG-prey (Faraji et al. 2000). The patterns of distribution of adults and eggs may therefore arise from a tendency to reduce intraguild interactions and this may increase opportunities for the coexistence of intraguild predators and prey in nature.

Acknowledgments $Y$. Choh received a scholarship from NWO (project 813.04.006). We thank Dr. Sven Bacher and two anonymous reviewers for their constructive comments.

Open Access This article is distributed under the terms of the Creative Commons Attribution Noncommercial License which permits any noncommercial use, distribution, and reproduction in any medium, provided the original author(s) and source are credited.

\section{References}

Agarwala BK, Yasuda H, Kajita Y (2003) Effect of conspecific and heterospecific feces on foraging and oviposition of two predatory ladybirds: role of fecal cues in predator avoidance. J Chem Ecol 29:357-376

Crawley MJ (2007) The R book. Wiley, New York

De Moraes GJ, McMurtry JA, Denmark HA, Campos CB (2004) A revised catalog of the mite family Phytoseiidae, 1st edn. Magnolia, Auckland

Faraji F, Janssen A, van Rijn PCJ, Sabelis MW (2000) Kin recognition by the predatory mite Iphiseius degenerans: discrimination among own, conspecific, and heterospecific eggs. Ecol Entomol 25:147-155

Fretwell SD (1972) Populations in a seasonal environment. Princeton University Press, Princeton

Fretwell SD, Lucas HL (1970) On territorial behavior and other factors influencing habitat distribution in birds. Acta Biotheor 19:16-36

Heithaus MR (2001) Habitat selection by predators and prey in communities with asymmetrical intraguild predation. Oikos 92:542-554

HilleRisLambers R, Dieckmann U (2003) Competition and predation in simple food webs: intermediately strong trade-offs maximize coexistence. Proc Biol Sci 270:2591-2598

Holt RD, Polis GA (1997) A theoretical framework for intraguild predation. Am Nat 149:745-764

Janssen A, Bruin J, Jacobs G, Schraag R, Sabelis MW (1997) Predators use volatiles to avoid prey patches with conspecifics. J Anim Ecol 66:223-232
Janssen A, Sabelis MW, Magalhães S, Montserrat M, van der Hammen T (2007) Habitat structure affects intraguild predation. Ecology 88:2713-2719

Lima SL (1998) Stress and decision making under the risk of predation: recent developments from behavioral, reproductive, and ecological perspectives. Adv Study Behav 27:215-290

Lima SL, Dill LM (1990) Behavioral decisions made under the risk of predation: a review and prospectus. Can J Zool 68:619-640

Magalhães S, Tudorache C, Montseratt M, van Maanen R, Sabelis MW, Janssen A (2005) Diet of intraguild predators affects antipredator behavior in intraguild prey. Behav Ecol 16:364-370

Montserrat M, Janssen A, Magalhães S, Sabelis MW (2006) To be an intraguild predator or a cannibal: is prey quality decisive? Ecol Entomol 31:430-436

Montserrat M, Bas C, Magalhães S, Sabelis MW, de Roos AM, Janssen A (2007) Predators induce egg retention in prey. Oecologia 150:699-705

Montserrat M, Magalhães S, Sabelis MW, de Roos AM, Janssen A (2008) Patterns of exclusion in an intraguild predator-prey system depend on initial conditions. J Anim Ecol 77:624-630

Mylius SD, Klumpers K, de Roos AM, Persson L (2001) Impact of intraguild predation and stage structure on simple communities along a productivity gradient. Am Nat 158:259-276

Nakashima Y, Birkett MA, Pye BJ, Powell W (2006) Chemically mediated intraguild predator avoidance by aphid parasitoids: interspecific variability in sensitivity to semiochemical trails of ladybird predators. J Chem Ecol 32:1989-1998

Persons MH, Rypstra AL (2001) Wolf spiders show graded antipredator behavior in the presence of chemical cues from different sized predators. J Chem Ecol 27:2493-2504

Persson L, Eklov P (1995) Prey refuges affecting interactions between piscivorous perch and juvenile perch and roach. Ecology $76: 70-81$

Polis GA, Holt R (1992) Intraguild predation: the dynamics of complex trophic interactions. Trends Ecol Evol 7:151-154

Polis GA, Winemiller KO (1996) Food webs. Chapman \& Hall, New York

Polis GA, Myer CA, Holt RD (1989) The ecology and evolution of intraguild predation: potential competitors that eat each other. Annu Rev Ecol Syst 20:297-330

R Development Core Team (2008) R: a language and environment for statistical computing. R Foundation for Statistical Computing, Vienna, Austria

Rosenheim JA, Kaya HK, Ehler HE, Marois JJ, Jaffee BA (1995) Intraguild predation among biological control agents-theory and evidence. Biol Control 5:303-335

Rypstra AL, Schmidt JM, Reif BD, DeVito J, Persons MH (2007) Tradeoffs involved in site selection and foraging in wolf spider: effects of substrate structure and predation risk. Oikos 116:853-863

Sih A (1998) Game theory and predator-prey response race. In: Dugatkin LA, Reeve HK (eds) Advances in game theory and study of animal behavior. Oxford University Press, Oxford, pp 221-238

Stephens D, Krebs JR (1986) Foraging theory. Princeton University Press, Princeton

van Rijn PCJ, Tanigoshi LK (1999) Pollen as food source for the predatory mites Iphiseius degenerans and Neoseiulus cucumeris (Acari: Phytoseiidae): dietary range and life history. Exp Appl Acarol 23:785-802

Venzon M, Janssen A, Pallini A, Sabelis MW (2000) Diet of a polyphagous arthropod predator affects refuge seeking of its thrips prey. Anim Behav 60:369-375

Wilder SM, Rypstra AL (2004) Chemical cues from an introduced predator (Mantodea, Mantidae) reduce the movement and foraging of a native wolf spider (Araneae, Lycosidae) in the laboratory. Environ Entomol 33:1032-1036 